\title{
LAS CAJAS DE AHORROS EN EL SISTEMA FINANCIERO EN ANDALUCIA
}

\author{
Cristina CAMPAYO RODRIGUEZ*
}

\section{INTRODUCCION}

El presente trabajo debe iniciarse señalando que la elección desu título no esen modo alguno arbitraria. Por el contrario, subraya una de las características más importantes que deben tenerse en cuenta cuando se analizan aspectos y problemas del sistema financiero a escala regional. En síntesis, sobre lo que se quiere llamar la atención es sobre el hecho de que no cabe hablar de un sistema financiero «de» una determinada zona geográfica del país, sino de las características de un sistema financiero común «en» una determinada región del mismo. Por ello, el título es el indicado, «las cajas de ahorros en el sistema financiero en Andalucía», y no el que sería, quizás, más estético literariamente hablando, pero incorrecto desde el punto de vista económico cual el de las cajas de ahorros en el sistema financiero andaluz.

Lo anterior implica, en primer lugar, la ausencia de sistemas financieros locales independientes y la existencia de relaciones financieras entre las diferentes zonas geográficas del país con unas características distintas de las que se producirían entre sistemas financieros independientes. En segundo lugar, la existencia de un elevado grado de subordinación a decisiones económicas y políticas de alcance más general sobre la asignación sectorial y territorial de los propios recursos financieros regionales. Y por último, un alto grado de homogeneidad y de adaptación de los instrumentos financieros a los usos generales del país y, más específicamente, al cuadro global definido por la legalidad vigente (instrumentos de captación de pasivos, fórmulas de financiación, condiciones de competitividad entre las diferentes instituciones crediticias, etc.) ${ }^{1}$.

* Profesora Titular de Economía Aplicada de la Universidad de Sevilla.

1. LAGARES CALVO, M.J.: Aspectos peculiares del sistema financiero en Andalucta. Economías de las Comunidades Autónomas: Andalucía. Papeles de Economía Española, págs. 187209, cit. pág. 187. 
Hecha esta importante observación general y antes de comenzar el análisis de las magnitudes más significativas que ponen de manifiesto la situación de las cajas de ahorros en el sistema financiero en Andalucía, parece oportuno comentar, brevemente, la metodología seguida en la ejecución del trabajo.

Para que el contenido del estudio responda a su título la investigación se ha realizado manejando un doble elemento de referencia, en virtud del cual las magnitudes se expresan con una dimensión temporal y comparada. De un lado, los cuadros elaborados contienen no sólo los datos de las cajas de ahorros sino también los correspondientes a la banca privada y las cajas rurales. De otro, la información que se maneja tiene un horizonte temporal de siete años, con la finalidad de apreciar con mayor rigor el papel que las cajas de ahorros han jugado y juegan en el sistema financiero en Andalucía.

\section{LOS DEPOSITOS DEL SISTEMA FINANCIERO EN ANDALUCIA}

El cuadro I contiene la información relativa al nivel de depósitos totales en el sistema financiero en Andalucía expresados en pesetas constantes de 1980. La característica fundamental de la evolución real de estos depósitos es su reducido ritmo de crecimiento interanual, sobre todo en el período que va desde 1982 a 1985. Pero estas cifras muestran también cómo en los dos últimos ejercicios contemplados (destacándose, sobre todo, el de 1987) el sistema financiero en Andalucía ha incrementado su captación de recursos.

\section{CUADRO I}

DEPOSITOS TOTALES EN EL SISTEMA FINANCIERO EN ANDALUCIA (Miles de millones de pesetas)

\begin{tabular}{|c|c|c|c|}
\hline Años & & P. constante de 1980 & $\begin{array}{c}\text { Tasa de variación } \\
\text { s/año anterior }\end{array}$ \\
\hline 1981 & 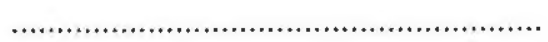 & $1.172,1$ & - \\
\hline 1982 & 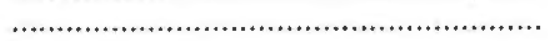 & $1.203,7$ & 2,69 \\
\hline 1983 & . & $1.210,0$ & 0,53 \\
\hline 1984 & 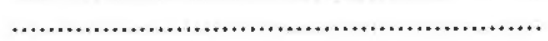 & $1.234,1$ & 1,99 \\
\hline 1985 & 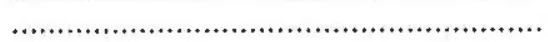 & $1.248,4$ & 1,16 \\
\hline 1986 & (1, & $1.277,1$ & 2,30 \\
\hline 1987 & 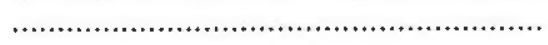 & $1.410,0$ & 10,41 \\
\hline
\end{tabular}

Fuente: Banco de España. Boletín Estadístico. Elaboración propia. 
Como muestra el cuadro II, los depósitos totales en la banca privada, en las cajas de ahorros y en las cajas rurales en Andalucía se nutren fundamentalmente de los depósitos realizados por el sector privado; las cuentas de organismos y corporaciones públicas tienen un papel muy secundario en la captación de fondos de estas instituciones financieras, aunque, sin embargo, han aumentado su participación en los años que se analizan. Por este motivo, en los epígrafes posteriores, el campo de estudio se va a reducir a los depósitos del sector privado, centrándose en el examen de los correspondientes a las cajas de ahorros.

\section{CUADRO II}

ESTRUCTURA DE LOS DEPOSITOS TOTALES EN EL SISTEMA FINANCIERO EN ANDALUCIA (En porcentajes)

\begin{tabular}{|c|c|c|c|}
\hline Años & & Sector público & Sector privado \\
\hline 1981 & 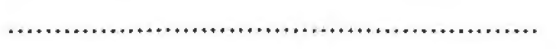 & 1,85 & 98,14 \\
\hline 1982 & 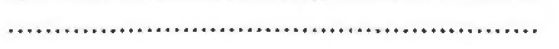 & 1,64 & 98,35 \\
\hline 1983 & 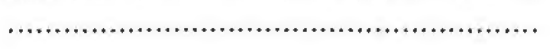 & 2,91 & 97,08 \\
\hline 1984 & 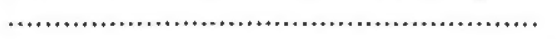 & 3,32 & 96,67 \\
\hline 1985 & (1) & 4,29 & 95,70 \\
\hline 1986 & . & 3,19 & 96,80 \\
\hline 1987 & 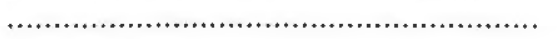 & 3,59 & 96,40 \\
\hline
\end{tabular}

Elaboración propia.

\subsection{Los depósitos del sector privado en las cajas de ahorros}

Como puede comprobarse en el cuadro III, los depósitos del sector privado en el sistema financiero en Andalucía han experimentado en estos años un crecimiento real muy moderado, incluso con pérdida de valor real en 1983. Pero hay que señalar que la tónica de incremento interanual de estos depósitos, al igual que la mostrada por los depósitos totales, cambia de ritmo en los dos últimos años.

El aspecto más importante a destacar de la información contenida en el cuadro III es el relativo a las diferencias que se han producido entre las evoluciones de los depósitos del sector privado captados por las distintas instituciones que conforman el sistema financiero. Así la banca privada ha perdido en términos reales recursos ajenos procedentes del sector privado en Andalucía en los años 1983 a 1985. Las cajas rurales sólo los perdieron en 1982 y los han ido incrementando a unas buenas tasas anuales en el resto de los ejercicios del período. Por el contrario, y como nota favorable, las cajas de ahorros han 


\section{CUADRO III}

DEPOSITOS DEL SECTOR PRIVADO EN EL SISTEMA FINANCIERO EN ANDALUCIA (Miles de millones de pesetas constantes de 1980)

\begin{tabular}{lcc}
\hline Años & P. constantes & $\begin{array}{c}\text { Tasa de variación } \\
\text { s/año anterior }\end{array}$ \\
\hline
\end{tabular}

Sistema bancario

\begin{tabular}{|c|c|c|c|}
\hline 1981 & , & $1.150,4$ & - \\
\hline 1982 & , & $1.183,9$ & 2,91 \\
\hline 1983 & . & $1.174,7$ & $-0,77$ \\
\hline 1984 & 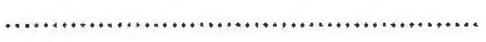 & $1.193,0$ & 1,55 \\
\hline 1985 & (n) & $1.194,8$ & 0,15 \\
\hline 1986 & . & $1.236,4$ & 3,55 \\
\hline 1987 & 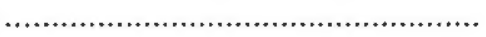 & $1.359,3$ & 9,94 \\
\hline
\end{tabular}

Cajas de Ahorros

\begin{tabular}{|c|c|c|c|}
\hline 1981 & & 363,2 & - \\
\hline 1982 & ....................... & 387,9 & 6,80 \\
\hline 1983 & .................. & 422,4 & 8,89 \\
\hline 1984 & $\ldots \ldots \ldots \ldots \ldots \ldots$ & 444,6 & 5,25 \\
\hline 1985 & . & 497,7 & 11,94 \\
\hline 1986 & $\ldots \ldots \ldots \ldots \ldots \ldots \ldots+\ldots$ & 517,2 & 3,91 \\
\hline 1987 & (2) & 575,5 & 11,27 \\
\hline
\end{tabular}

\section{Banca privada}

\begin{tabular}{|c|c|c|c|}
\hline 1981 & (1) & 706,1 & - \\
\hline 1982 & , & 718,2 & 1,71 \\
\hline 1983 & ............... & 670,8 & $-6,59$ \\
\hline 1984 & (n....................... & 663,8 & $-1,04$ \\
\hline 1985 & …........... & 603,4 & $-9,09$ \\
\hline 1986 & . & 623,6 & 3,34 \\
\hline 1987 & …............................. & 677,4 & 8,62 \\
\hline
\end{tabular}

Cajas Rurales

\begin{tabular}{|c|c|c|c|}
\hline 1981 & 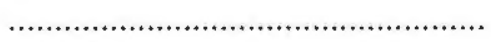 & 81,0 & 一 \\
\hline 1982 & ..... & 77,8 & $-3,95$ \\
\hline 1983 & . & 81,4 & 4,62 \\
\hline 1984 & . & 84,5 & 3,80 \\
\hline 1985 & 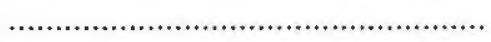 & 93,6 & 10,76 \\
\hline 1986 & (.)..................................... & 95,5 & 2,02 \\
\hline 1987 & (n) & 106,3 & 11,30 \\
\hline
\end{tabular}

Fuente: Banco de España. Boletín Estadístico. Elaboración propia. 
mostrado capacidad no sólo para mantener el valor real de los depósitos que en ellas impone el sector privado, sino para aumentarlos progresivamente a un ritmo importante.

Un punto fundamental para el análisis de los depósitos del sector privado en las cajas de ahorros en Andalucía es el conocimiento de la estructura institucional de los citados depósitos, ya que ello resulta de interés para valorar el papel que tales entidades vienen jugando, y pueden jugar, en el futuro en el sistema financiero así como en la financiación de la economía andaluza.

Los datos referentes a la estructura institucional de los depósitos del sector privado en Andalucía ponen claramente de manifiesto el peso creciente de las cajas de ahorros en el sistema financiero a este nivel regional. La evolución descrita de la captación de recursos ajenos en el sector privado por parte de estas instituciones es la que les ha permitido que en 1987 hayan alcanzado una posición más paritaria con la banca privada; y de continuar esta tónica estas entidades llegarán a tener cuotas de mercado similares.

En el cuadro IV también se aprecia la constancia de los valores de la cuota de mercado relativa a depósitos de las cajas rurales, así como el retroceso que la banca privada está experimentando en su captación de estos recursos ajenos en Andalucía. Los incrementos producidos en los depósitos que el sector privado ha realizado en estas entidadses han tenido como destino, fundamentalmente, las cajas de ahorros.

\section{CUADRO IV}

ESTRUCTURA INSTITUCIONAL DE LOS DEPOSITOS DEL SECTOR PRIVADO EN ANDALUCIA (En porcentajes)

\begin{tabular}{|c|c|c|c|c|}
\hline Años & & Banca privada & Cajas de Ahorros & Cajas Rurales \\
\hline 1981 & (n............ & 61,37 & 31,57 & 7,04 \\
\hline 1982 & 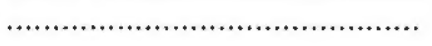 & 60,66 & 32,76 & 6,57 \\
\hline 1983 & 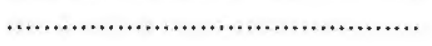 & 57,10 & 35,96 & 6,93 \\
\hline 1984 & 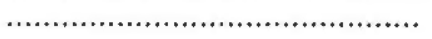 & 55,63 & 37,27 & 7,08 \\
\hline 1985 & , & 50,50 & 41,65 & 7,84 \\
\hline 1986 & . & 50,43 & 41,83 & 7,72 \\
\hline 1987 & 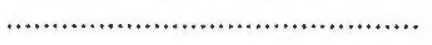 & 49,83 & 42,33 & 7,82 \\
\hline
\end{tabular}

Elaboración propia.

Para completar este análisis, y apreciar con más claridad la verdadera connotación que el sistema financiero tiene en Andlaucía, se añade, en el cuadro $\mathrm{V}$, la estructura institucional de los depósitos del sector privado a nivel nacional. Esta información, junto con la contenida en el cuadro IV, nos muestra el papel especialmente importante que en Andalucía tienen las cajas rurales, 
hasta el punto de que los depósitos en ellas efectuados supusieron en estos años casi la cuarta parte del total nacional ${ }^{2}$. Es evidente que este importante papel de las cajas rurales andaluzas, que lo han tenido desde años anteriores a los que estudiamos aquí, viene en buena medida determinado por una estructura económica en la que el sector agrario desempeña un papel asimismo muy superior al que ejerce, por témino medio, en el resto de España.

\section{CUADRO V}

ESTRUCTURA INSTITUCIONAL DE LOS DEPOSITOS DEL SECTOR PRIVADO A NIVEL NACIONAL

(En porcentajes)

\begin{tabular}{|c|c|c|c|c|}
\hline Años & & Banca privada & Cajas de Ahorros & Cajas Rurales \\
\hline 1981 & $\ldots$ & 63,57 & 33,41 & 3,01 \\
\hline 1982 & 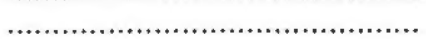 & 63,20 & 33,70 & 3,09 \\
\hline 1983 & 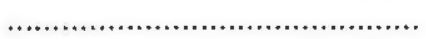 & 61,68 & 35,08 & 3,23 \\
\hline 1984 & 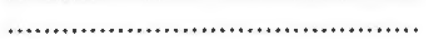 & 60,94 & 35,66 & 3,39 \\
\hline 1985 & , & 56,72 & 39,41 & 3,86 \\
\hline 1986 & 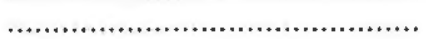 & 56,67 & 39,71 & 3,61 \\
\hline 1987 & , & 56,59 & 39,02 & 4,37 \\
\hline
\end{tabular}

Fuente: Banco de España. Boletín Estadístico. Elaboración propia.

Si nos fijamos en el peso respectivo que han alcanzado las cajas de ahorros y la banca privada en el sistema financiero a nivel nacional y en Andalucía, vemos que en estos años los correspondientes a las primeras instituciones mencionadas han ido progresivamente elevándose y lógicamente los relativos a las segundas se han ido reducido de manera continuada. Luego, como es evidente, la banca privada esta perdiendo cuota de mercado en su actividad de captación de fondos en el sector privado, mientras que las cajas de ahorros la están ganando paulatinamente.

La situación comentada ha sido más favorable para las cajas de ahorros en el sistema financiero en Andalucía, pues de un peso inicial en 1981 del 31,57 $\%$, inferior al del $33,41 \%$ que presentaban en el conjunto nacional, han conseguido uno final del $42,33 \%$ en 1987 , que es superior en 3,31 puntos al que alcanzan las cajas de ahorros en el sistema financiero de la nación.

En un estudio realizado recientemente sobre las cajas de ahorros españolas ${ }^{3}$, se demuestra que la década que discurre entre 1976 y 1986 ha sido un período excepcional en la vida de estas instituciones. Tanto por los profundos cambios

2. Ibidem, págs. 194-195.

3. LAGARES CALVO, M.J.: Los resultados de las Cajas de Ahorros. Papeles de Economía Española n, ${ }^{\circ} 32,1987$, págs. 292 a 313, cit. pág. 157-159. 
que han experimentado en su configuración y actividades, como, en otro orden de cosas, por su afianzamiento como entidades de depósitos, lo que se plasma en el crecimiento experimentado en la cuota de mercado de sus depósitos en el sistema financiero nacional. Como antes se ha puesto de manifiesto este afianzamiento de las cajas de ahorros como entidades de depósitos ha adquirido una mayor relevancia en el sistema financiero en Andalucía.

\subsection{Estructura funcional de los depósitos del sector privado en las cajas de ahorros}

En el cuadro VI se recoge la estructura funcional de depósitos atendiendo al plazo de su colocación para los años 1983 a 1987. El período ha tenido que reducirse en relación al que se ha manejado hasta el momento y se ha debido, asimismo, prescindir de los datos relativos a las cajas rurales por no facilitar los Boletines Estadísticos del Banco de España la información necesaria para ello.

\section{CUADRO VI}

ESTRUCTURA FUNCIONAL DE LOS DEPOSITOS DEL SECTOR PRIVADO EN EL SISTEMA FINANCIERO EN ANDALUCIA

(En porcentajes)

\begin{tabular}{|c|c|c|c|c|}
\hline Años & & D. a la vista & D. de ahorro & D. a plazo \\
\hline \multicolumn{5}{|c|}{ Cajas de Ahorros } \\
\hline 1983 & 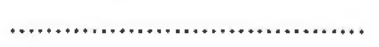 & 11,96 & 52,11 & 35,66 \\
\hline 1984 & & 12,87 & 49,56 & 37,54 \\
\hline 1985 & , & 12,95 & 46,66 & 40,38 \\
\hline 1986 & 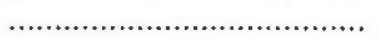 & 12,60 & 46,19 & 41,19 \\
\hline 1987 & 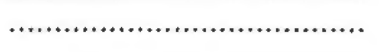 & 16,11 & 51,30 & 47,42 \\
\hline \multicolumn{5}{|c|}{ Banca privada } \\
\hline 1983 & ......................... & 24,56 & 16,80 & 58,55 \\
\hline 1984 & 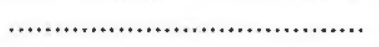 & 27,41 & 16,50 & 56,08 \\
\hline 1985 & 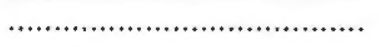 & 30,40 & 18,43 & 51,15 \\
\hline 1986 & 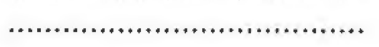 & 28,56 & 18,12 & 53,31 \\
\hline 1987 & 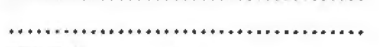 & 27,84 & 17,14 & 54,99 \\
\hline
\end{tabular}

Fuente: Banco de España. Boletín Estadístico. Elaboración propia.

A la vista del cuadro VI cabe hacer una primera observación de carácter general cual es la de la diferente estructura funcional de los depósitos del sector privado de Andalucía en las cajas de ahorros y en la banca privada. Esta diferente estructura se manifiesta, en primer lugar, en el peso superior que 
tanto los depósitos a la vista como a plazo tienen en la banca privada en relación al que alcanzan en las cajas de ahorros, aunque esas diferencias de pesos han ido reduciéndose a lo largo del período; en segundo lugar, en que la diferencia más acusada entre estas estructuras funcionales se encuentra en los pesos de los depósitos de ahorros, pues a éstos se les puede calificar para la banca privada como la modalidad residual de captación de fondos, mientras que para las cajas de ahorros hay que calificarlos como la principal fuente de obtención de recursos ajenos.

La liberalización de retribuciones para una parte del pasivo de las entidades financieras, iniciada en 1977 y continuada posteriormente, al permitir una libertad completa en las retribuciones satisfechas a los depósitos a plazo superior a seis meses, ha provocado una diferenciación de recursos en dos bloques: los que podrían denominarse baratos, que han coincidido con los depósitos intervenidos hasta hace muy poco, es decir, las cuentas corrientes, las cuentas de ahorro a la vista y los depósitos a plazo hasta seis meses; y los recursos caros, representados por los de plazos superiores a seis meses así como otras formas de captación de fondos (cédulas hipotecarias, empréstitos y cesiones temporales de activos).

Pues bien, en los años objeto de análisis, las cajas de ahorros en Andalucía han obtenido sus recursos, fundamentalmente, a través de las colocaciones que se pueden denominar baratas. Aunque también se ha producido un cierto desplazamiento de su obtención de fondos mediante depósitos que en principio se pueden calificar de caros si superan el plazo de seis meses, ya que se ha incrementado progresivamente el peso de las imposiciones a plazo en la estructura funcional de sus depósitos.

En el cuadro VII se recoge la estructura de depósitos atendiendo a su plazo de colocación en el sistema financiero a nivel nacional. Se ha introducido esta información con el objeto de compararla con la comentada estructura funcional de depósitos en las cajas de ahorros y en la banca privada en Andalucía. De esta comparación se desprende lo siguiente:

- La estructura funcional de los depósitos en Andalucía se ha separado, entre 1983 y 1987, de los valores de la estructura funcional de depósitos de las instituciones financieras a nivel nacional.

- Los depósitos a la vista tienen un peso relativo superior en Andalucía, tanto en las cajas de ahorros como en la banca privada, al que cada una de estas entidades ostentan en el conjunto nacional.

- Los depósitos de ahorro no presentan a nivel nacional la importancia que, como forma de obtención de recursos ajenos, tienen en las cajas de ahorros en Andalucía; esta modalidad de depósito es, además, más 
utilizada por el sector privado en la región que nos ocupa que en el conjunto de regiones españolas, pues estos depósitos en la banca privada en Andalucía adquieren una importancia que no alcanzan en estas instituciones a nivel nacional.

- Los depósitos a plazo, en cambio, han constituído, tanto en las cajas de ahorros como en la banca privada a nivel nacional, la principal fuente de obtención de recursos, mientras que no es ésta la situación que se produce en estas entidades en Andalucía, como se ha comprobado, constituyendo los depósitos a plazo la segunda vía de captación de fondos.

\section{CUADRO VII}

ESTRUCTURA FUNCIONAL DE LOS DEPOSITOS DEL SECTOR PRIVADO EN EL SISTEMA FINANCIERO A NIVEL NACIONAL

(En porcentajes)

\begin{tabular}{|c|c|c|c|c|}
\hline Años & & D. a la vista & D. de ahorro & D. a plazo \\
\hline \multicolumn{5}{|c|}{ Cajas de Ahorros } \\
\hline 1983 & 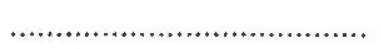 & 10,66 & 43,95 & 45,38 \\
\hline 1984 & 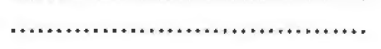 & 11,10 & 42,90 & 45,99 \\
\hline 1985 & (n) & 11,71 & 41,66 & 46,61 \\
\hline 1986 & 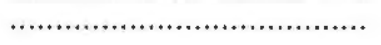 & 12,04 & 41,51 & 46,43 \\
\hline 1987 & 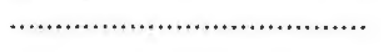 & 13,60 & 40,33 & 46,05 \\
\hline \multicolumn{5}{|c|}{ Banca privada } \\
\hline 1983 & …, & 23,24 & 13,54 & 63,20 \\
\hline 1984 & 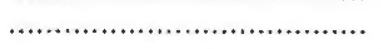 & 24,59 & 13,45 & 61,94 \\
\hline 1985 & (1) & 24,47 & 14,82 & 57,69 \\
\hline 1986 & (1) & 26,91 & 14,46 & 58,61 \\
\hline 1987 & 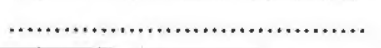 & 26,63 & 13,31 & 60,05 \\
\hline
\end{tabular}

Fuente: Banco de España. Boletín Estadístico. Elaboración propia.

\subsection{La expansión de las cajas de ahorros}

Para analizar cómo se ha producido la expansión de las cajas de ahorros en Andalucía en los años que nos ocupan, resultaría necesario analizar la política seguida por estas entidades en relación a la apertura de nuevas oficinas y al cierre de otras existentes. Pero una vez más la falta de información no permite efectuar este análisis. Esta laguna informativa se puede suplir en parte conociendo el número de oficinas con el que cuentan las cajas de ahorros en Andalucía, así como su situación en la red de sucursales de las entidades de depósitos en esta comunidad autónoma. 
El cuadro VIII contiene el número de oficinas que en 1984 tenían abiertas las instituciones financieras en Andalucía y a nivel nacional. Las cajas de ahorros contaban con 1.602 oficinas funcionando en la región, frente a las 1.890 oficinas de la banca privada y a las 632 de las cajas rurales. A nivel nacional es, también, la banca privada la que tiene abiertas un mayor número de oficinas, siguiéndola las cajas de ahorros y las cajas rurales.

CUADRO VIII

NUMERO DE OFICINAS DE LAS INSTITUCIONES FINANCIERAS EN 1984

\begin{tabular}{|c|c|c|c|c|}
\hline & \multicolumn{2}{|c|}{ Número de oficinas } & \multicolumn{2}{|c|}{$\begin{array}{l}\text { Depósitos por oficinas } \\
\text { (en miles de ptas.) }\end{array}$} \\
\hline & Andalucía & España & Andalucía & España \\
\hline Banca privada & 1.890 & 16.412 & 552,5 & 713,3 \\
\hline Cajas de Ahorros ................... & 1.602 & 10.440 & 436,7 & 656,3 \\
\hline Cajas Rurales . ....................... & 632 & 3.315 & 210,4 & 196,5 \\
\hline
\end{tabular}

Fuente: Banco de España. Boletín Estadístico. Elaboración propia.

Aunque en el «ranking» del número de oficinas, al igual que en el del volumen de depósitos, las cajas de ahorros se sitúen por debajo de la banca privada, el dato que nos pone de manifiesto la forma de actuar de las primeras es el volumen de depósitos captados por oficina. En el mismo cuadro VIII se comprueba cómo las cajas de ahorros, y de una forma más acusada en Andalucía, han captado un menor volumen de depósitos por oficina que la banca privada.

La información que facilita el cuadro es fácil de prever, pues, aun cuando la financiación de las grandes empresas y del sector público o la participación cada vez más intensa en las actividades de los mercados financieros internacionales sean nuevos ámbitos de actividad que en los últimos tiempos han adquirido una considerable importancia en las actividades de las cajas de ahorros españolas, no cabe duda de que, en todo caso, siguen resultando complementarias de la actividad principal que estas entidades desarrollan: «la actividad financiera al por menor», como ha sido calificada ${ }^{4}$, orientada básicamente al servicio de las necesidades de los individuos, de las familias y de las empresas de dimensión media y pequeña.

Las actividades financieras al por menor obligan a estar presente en muchos puntos geográficos a la vez para captar y atender adecuadamente millones de pequeñas operaciones, y hacen que disminuya el volumen de negocio de cada

4. LAGARES CALVO, M.J.: Cajas de Ahorros: Los retos del futuro. Papeles de Economía Española n.o 36, 1988, págs. 157-172, cit. pág. 157-159. 
centro operativo. También hacen que resulte bastante más costoso, desde el punto de vista operativo, el desarrollo de tales actividades, aunque la automatización e informatización de los procesos esté permitiendo que los costes operativos unitarios se estén reduciendo de forma considerable en el ámbito de los servicios financieros al por menor.

El hecho de que las cajas de ahorros en Andalucía presenten un volumen de depósitos por oficina menor del que alcanzan estas entidades a nivel nacional se deriva de la propia estructura económica y grado de desarrollo de esta comunidad autónoma, donde abundan las actividades financieras al por menor, como lo demuestra el que la banca privada, también obtenga en Andalucía un menor volumen de depósitos por oficina que en el conjunto nacional.

\section{LA INVERSION CREDITICIA DEL SISTEMA FINANCIERO EN ANDALUCIA}

Es evidente que la funcionalidad de un sistema financiero - y más específicamente de un sistema crediticio- se halla en proporción directa a su eficiencia para captar recursos y actuar como intermediario para su colocación, de acuerdo con el abanico de posibilidades de endeudamiento de los diversos agentes que demandan medios financieros con que dotar sus actividades económicas.

Obviamente, los datos sobre los que debe apoyarse el conocimiento de la capacidad funcional de un sistema financiero no se refieren tan sólo al pasivo de la instituciones de crédito, sino que debe extenderse el conocimiento de la dinámica inversora de las mismas. Desgraciadamente, la información estadística disponible no se caracteriza ni por su extensión ni, en ocasiones, por su calidad. Este problema es más acusado en el caso de pretender adentrarse en el análisis a nivel regional, donde la escasez de estadísticas es más acusada y presenta barreras prácticamente insalvables en el aspecto financiero.

En la vertiente inversora de las instituciones financieras, los datos a nivel regional que pueden manejarse se refieren tan sólo a los créditos concedidos. Lógicamente ello implica que sólo podamos analizar uno de los componentes de la mencionada vertiente inversora, sin poder estudiar la política seguida por estas entidades en la inversión en valores. Y, además, los datos regionalizados de créditos otorgados por las entidades a que nos estamos refiriéndo sólo se han obtenido a partir de 1983 , por lo cual ha sido preciso, una vez más, acortar el período de análisis (cuadro IX).

La evolución real de la inversión crediticia del sistema financiero en Andalucía no se separa sensiblemente de la experimentada por los depósitos totales captados en esta región. La diferencia más apreciable se encuentra en hecho 
de que la evolución que ahora estamos analizando ha experimentado pérdidas de sus valores reales en dos ejercicios del período, adquiriendo una considerable importancia la correspondiente a 1984, pues la tasa anual que la mide se situa en el $-9,32 \%$.

\section{CUADRO IX}

LA INVERSION CREDITICIA DEL SISTEMA FINANCIERO EN ANDALUCIA (En millones de pesetas)

\begin{tabular}{|c|c|c|}
\hline Años & P. constantes & $\begin{array}{l}\text { Tasa de variación } \\
\text { s/año anterior }\end{array}$ \\
\hline 1983 & 840,6 & - \\
\hline 1984 & 762,2 & $-9,32$ \\
\hline 1985 & 758,6 & $-0,47$ \\
\hline 1986 & 812,3 & 7,07 \\
\hline 1987 2.0 T. & 884,1 & 8,83 \\
\hline
\end{tabular}

Fuente: Boletín Económico de Andalucía, n. ${ }^{0}$ 9, III y IV trimestre de 1987. Elaboración propia.

\subsection{La inversión crediticia de las cajas de ahorros}

El volumen de crédito concedido por las cajas de ahorros en Andalucía se muestra en el cuadro X. La evolución real de estos créditos no ha sido homogénea en el período; así en 1985 se produjo una pérdida de su valor real mientras que en los otros años aumentaron a unas buenas tasas que alcanzaron, incluso, unos niveles de crecimiento superiores a los conseguidos por los recursos ajenos captados por estas instituciones en la comunidad autónoma.

\section{CUADRO X}

LA INVERSION CREDITICIA DE LAS CAJAS DE AHORROS EN ANDALUCIA (En millones de pesetas)

\begin{tabular}{|c|c|c|}
\hline Años & P. constantes & $\begin{array}{c}\text { Tasa de variación } \\
\text { s/año anterior }\end{array}$ \\
\hline 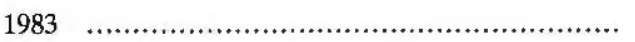 & 240,8 & - \\
\hline 1984 & 255,3 & 6,03 \\
\hline 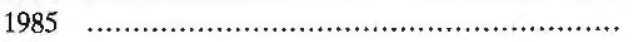 & 253,2 & $-0,78$ \\
\hline 1986 & 284,4 & 12,33 \\
\hline 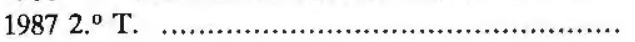 & 321,2 & 12,95 \\
\hline
\end{tabular}

Fuente: Boletín Económico de Andalucía, n. ํ 9, III y IV trimestre de 1987. Elaboración propia. 
En el cuadro XI se recoge la evolución real de la inversión crediticia efectuada tanto por la banca privada como por las cajas rurales en Andalucía. Si las comparamos con la evolución relativa a las cajas de ahorros (y con este fin se ha introducido la información) vemos cómo, en los años que analizamos, ha crecido a un ritmo anual más elevado el valor real de los créditos otorgados por las cajas de ahorros en esta región que los concedidos por las otras dos entidades.

Una conclusión que se desprende de lo que llevamos analizado es que las cajas de ahorros están aumentando, en estos años de la década de los ochenta, su captación de recursos y su concesión de créditos en Andalucía en mayor medida que la banca privada y las cajas rurales.

\section{CUADRO XI}

LA INVERSION CREDITICIA DE LA BANCA PRIVADA

Y LAS CAJAS RURALES EN ANDALUCIA

(En millones de pesetas)

\begin{tabular}{|c|c|c|c|c|}
\hline \multirow[b]{2}{*}{ Años } & \multicolumn{2}{|c|}{ Banca privada } & \multicolumn{2}{|c|}{ Cajas Rurales } \\
\hline & P. constantes & $\begin{array}{l}\text { Tasa variación } \\
\text { s/año anterior }\end{array}$ & P. constantes & $\begin{array}{l}\text { Tasa variación } \\
\text { s/año anterior }\end{array}$ \\
\hline $1983 \quad \ldots \ldots \ldots \ldots \ldots \ldots \ldots \ldots \ldots \ldots \ldots \ldots \ldots \ldots \ldots \ldots \ldots \ldots$ & 534,6 & - & 64,9 & - \\
\hline $1984 \quad \ldots \ldots \ldots \ldots \ldots \ldots \ldots \ldots \ldots \ldots \ldots \ldots \ldots \ldots \ldots \ldots \ldots$ & 452,5 & $-15,30$ & 54,2 & $-16,45$ \\
\hline 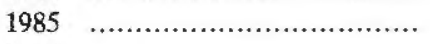 & 447,9 & $-1,00$ & 57,3 & 5,86 \\
\hline $1986 \quad \ldots \ldots \ldots \ldots \ldots \ldots \ldots \ldots \ldots \ldots \ldots \ldots \ldots \ldots \ldots \ldots \ldots$ & 468,4 & 4,59 & 59,2 & 3,48 \\
\hline 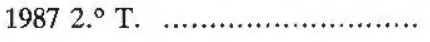 & 498,6 & 6,44 & 64,3 & 8,63 \\
\hline
\end{tabular}

Fuente: Boletín Económico de Andalucía, n. ${ }^{\circ}$ 9, III y IV trimestre de 1987. Elaboración propia.

El cuadro XII contiene la estructura institucional de la inversión crediticia en Andalucía. Los datos del cuadro ponen de manifiesto la afirmación efectua$\mathrm{da}$, pues el peso de las cajas de ahorros en esta vertiente inversora de las instituciones financieras en la región ha ido elevándose sucesivamente en el período, y es consecuencia fundamental de la evolución experimentada por la concesión de créditos de estas entidades.

Un hecho que hay que destacar en relación con el tema que ahora nos ocupa, es la mayor relevancia que la banca privada tiene en la inversión crediticia en Andalucía en comparación con la que alcanza en esos mismos años en la estructura institucional de depósitos en esta comunidad autónoma, aunque ambas posiciones han experimentado una evolución decreciente en el período.

Los coeficientes de inversión obligatoria más elevados que de antiguo han tenido que cumplir las cajas de ahorros, aunque unificados sus niveles a partir 
de $1985^{5}$, y el hecho de que porcentajes considerables de los mismos hayan tenido que materializarse en la adquisición de determinados títulos valores, ha determinado que estas instituciones tengan una menor importancia en la inversión crediticia en Andalucía que la que han conseguido alcanzar en la captación de recursos. No obstante, las sucesivas liberalizaciones, producidas en los últimos años por distintas vías, de los recursos afectados al cumplimiento de los coeficientes obligatorios de inversión, ha sido uno de los factores que ha contribuido al significativo crecimiento de la actividad de concesión de créditos que las cajas de ahorros muestran en los años que estudiamos.

CUADRO XII

ESTRUCTURA INSTITUCIONAL DE LA INVERSION CREDITICIA EN ANDALUCIA (En porcentajes)

\begin{tabular}{|c|c|c|c|}
\hline Años & Cajas de Ahorros & Banca privada & Cajas Rurales \\
\hline . & 23,65 & 63,59 & 7,72 \\
\hline 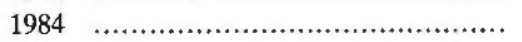 & 33,49 & 59,37 & 7,11 \\
\hline 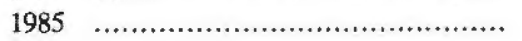 & 33,38 & 59,07 & 7,56 \\
\hline 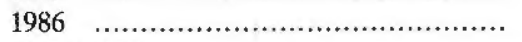 & 35,03 & 57,67 & 7,29 \\
\hline 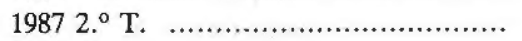 & 36,33 & 56,39 & 7,27 \\
\hline
\end{tabular}

Elaboración propia.

\subsection{Distribución sectorial de la inversión crediticia de las cajas de ahorros}

La distribución sectorial de la inversión crediticia de las cajas de ahorros en Andalucía se limita a la diferenciación entre los créditos destinados al sector público y al sector privado. La falta de estadísticas sobre la distribución de esta inversión entre las diferentes actividades económicas, imposibilita el conocer la contribución de las instituciones que analizamos a la financiación de las mencionadas actividades económicas en la región.

La concesión de créditos al sector público está adquiriendo una importancia creciente en la cartera de créditos de las cajas de ahorros en Andalucía, como lo demuestra el hecho de que en 1983 suponían el 4,96\% de estas inversiones y tan sólo tres años y seis meses después representaban ya el 7,59 \%. El crecimiento del volumen de los créditos otorgados al sector público ha coincidido

5. POVEDA ANADON, R.: El coeficiente de inversión 1985-1986. Papeles de Economía Española n. ${ }^{0} 32,1987$, págs. 396-410. 
con la consolidación de la comunidad autónoma andaluza y con el relanzamiento de las actividades de las corporaciones locales, lo que significa que las cajas de ahorros en Andalucía han atendido de una forma creciente a las necesidades financieras de los mencionados entes públicos autónomos y locales.

La presencia creciente que los créditos otorgados al sector público ha tenido, y presumiblemente tendrán en el futuro, en esta actividad inversora de las cajas de ahorros se deriva de la regulación legal del coeficiente de inversión obligatoria de estas entidades, ya que, pese a su reducción, dentro de lo que se consideran activos computables, los créditos a organismos públicos han adquirido un mayor peso ${ }^{6}$ (cuadro XIII).

\section{CUADRO XIII}

DISTRIBUCION SECTORIAL DE LA INVERSION CREDITICIA DE LAS CAJAS DE AHORROS EN ANDALUCIA

(En porcentajes)

\begin{tabular}{|c|c|c|}
\hline Años & Sector público & Sector privado \\
\hline 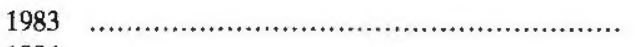 & 4,96 & 95,03 \\
\hline 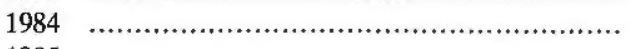 & 5,40 & 94,59 \\
\hline 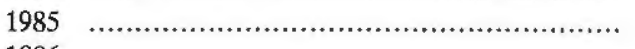 & 6,70 & 93,29 \\
\hline 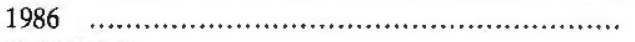 & 6,29 & 93,70 \\
\hline 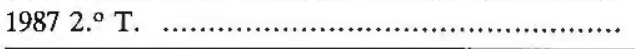 & 7,59 & 92,40 \\
\hline
\end{tabular}

Fuente: Boletín Económico de Andalucía, n. ${ }^{\circ}$ 9, III y IV trimestre de 1987. Elaboración propia.

\section{CUADRO XIV}

DISTRIBUCION SECTORIAL DE LA INVERSION CREDITICIA DE LA BANCA PRIVADA EN ANDALUCIA

(En porcentajes)

\begin{tabular}{|c|c|c|}
\hline Años & Sector público & Sector privado \\
\hline (n........................ & 1,09 & 98,90 \\
\hline 1984 & 1,19 & 98,80 \\
\hline 1985 & 1,73 & 98,26 \\
\hline 1986 & 1,97 & 98,02 \\
\hline $198720^{\circ} \mathrm{T}$. & 2,30 & 97,69 \\
\hline
\end{tabular}

Fuente: Boletín Económico de Andalucía, n. ${ }^{\circ}$ 9, III y IV trimestre de 1987. Elaboración propia.

6. En el período que comprende la investigación, el texto legal más significativo ha sido la Ley $13 / 1985$, de 25 de mayo, de coeficientes de inversión, recursos propios y obligaciones de información de los intermediarios financieros. 
Lo que se acaba de señalar viene confirmado, por una parte, por la distribución sectorial de la inversión crediticia de la banca privada en Andalucía (cuadro XIV) donde los créditos al sector público adquieren un menor peso en su cartera de créditos que el que presentan los otorgados por las cajas ahorros en su correspondiente cartera. Y por otra, el hecho de que el sector público ha obtenido unos mayores volúmenes de financiación en las cajas de ahorros que en la banca privada en Andalucía.

\section{CONCLUSIONES}

A lo largo del trabajo que se acaba de exponer se pone de manifiesto que las cajas de ahorro han ido adquiriendo, en los últimos años, un papel creciente y cada vez más relevante en el sistema financiero en Andalucía. Lo que se evidencia en el crecimiento tanto de su capacidad de captación de recursos como en su actividad crediticia en la región. Ello las ha llevado a alcanzar unos niveles en sus cuotas de mercado muy similares a los de la banca privada. Este fenómeno se ha producido a la par que ha tenido lugar un retroceso del papel que la banca privada venía desempeñando, por lo que puede decirse que ambas circunstancias están concatenadas, de nianera que el incremento de la relevancia de las cajas de ahorros ha sido posible a costa de una disminución de la importancia relativa de la banca privada.

La vocación regional que han demostrado las cajas de ahorros en Andalucía y su afianzamiento como entidades de depósitos y de créditos en esta región, de mantenerse, debe llevarlas a incrementar aún más la actividad principal que estas instituciones desarrollan (las denominadas operaciones «al por menor»), y que en la comunidad autónoma andaluza por sus características económicas encuentra una gran acogida. Pero esto no debe hacer olvidar el surgimiento y desarrollo de nuevos ámbitos de actuación de las cajas de ahorros (financiación de las grandes empresas y del sector público así como una participación más intensa en los mercados financieros internacionales) fundamentales para que las cajas de ahorros puedan competir con el resto de las instituciones financieras, tanto las ya existentes como las que en el futuro se establezcan, sobre todo teniendo en cuenta el marco del Mercado Unico Europeo.

Los recursos liberados, y los que continuarán liberándose, por las sucesivas reducciones del coeficiente de inversión obligatoria deben ser, por otra parte, destinados por las cajas de ahorros a la financiación del proceso de modernización y transformación de la economía andaluza, pues su contribución al mismo, que las llevará a participar en actividades y a acoger a segmentos de clientes que tradicionalmente no constituían la clientela fundamental de estas entidades, será un elemento fundamental para adquirir un papel primordial en el sistema financiero en Andalucía. 
No quiero acabar este trabajo sin hacer mención al tema de la posible fusión o fusiones de todas o de algunas cajas de ahorros andaluzas. Como es sabido, el proceso de concentración de entidades bancarias ocupa un lugar primordial en la atención pública, debido a que en 1992 se pone en marcha el Mercado Unico Europeo, como se ha señalado anteriormente, y por consiguiente habrá plena libertad de movimiento de capitales y de establecimiento de entidades financieras entre los Estados miembros de la CEE.

Si a la dimensión de las entidades bancarias españolas se la califica de pequeña en comparación con las del resto de Europa, y preocupa, pues, la posición competitiva de la banca española y la posibilidad de que ésta pueda ser absorbida por la banca extranjera en el proceso de integración económica europea $^{7}$, de más pequeña hay que calificar a la dimensión de las distintas cajas de ahorros andaluzas y más preocupante es su posición competitiva. Por lo tanto un proceso de fusión o de fusiones entre estas instituciones, en mi opinión, contribuiría al menos:

- A aprovechar las economías de escala existentes.

- A atender las necesidadses de crédito de los grandes clientes.

- A una racionalización de la red de sucursales.

- A adquirir una mejor posición para competir con las entidades bancarias extranjeras que se establezcan en nuestro país.

Pero no se puede olvidar que las fusiones, en el mejor de los casos constituyen un proceso lento y no falto de costes y dificultades derivadas, entre otras causas, de la necesidad de combinar las plantillas de las diferentes instituciones, adecuar la red de sucursales y armonizar la red de clientes ${ }^{8}$.

7. VIVES, X.: Concentración bancaria y competitividad. Papeles de Economía Española n. ${ }^{\circ} 36$, 1988, págs. 62-75, cit. pág. 62.

8. REVELL, J.: Las fusiones y el papel de los grandes bancos. Papeles de Economía Española n. ${ }^{\circ} 36,1988$, págs. $90-112$, cit. págs. 111-112.

NOTAS A LOS CUADROS: 1) El deflactor que se ha utilizado ha sido el deflactor implícito en el PIB a precios de mercado tomando como base el año 1980. 2) Los cuadros referentes a los depósitos, hasta el año 1982 inclusive, no incluyen los depósitos en moneda extranjera ni los depósitos de las cajas de ahorros en la banca privada. A partir de 1983 se incluyen los depósitos ordinarios, las cuentas de ahorro del emigrante y los depósitos en moneda extranjera. 3) Los cuadros referentes a la inversión crediticia están elaborados sobre la base de los datos contenidos en el Boletín Económico de Andalucía, que, a su vez, los ha obtenido del Banco de España; pero son cifras provisionales que no constan aún en el Boletín Estadístico del Banco de España. 